Voix et Images

\title{
La culture savante en Nouvelle-France : les écrits de Pierre Potier (1708-1781)
}

\section{Caroline Masse}

Volume 22, numéro 3 (66), printemps 1997

Gilbert Langevin

URI : https://id.erudit.org/iderudit/201334ar

DOI : https://doi.org/10.7202/201334ar

Aller au sommaire du numéro

Éditeur(s)

Université du Québec à Montréal

ISSN

0318-9201 (imprimé)

1705-933X (numérique)

Découvrir la revue

Citer cet article

Masse, C. (1997). La culture savante en Nouvelle-France : les écrits de Pierre Potier (1708-1781). Voix et Images, 22(3), 629-633.

https://doi.org/10.7202/201334ar d'utilisation que vous pouvez consulter en ligne.

https://apropos.erudit.org/fr/usagers/politique-dutilisation/ 
Recherche

\section{La culture savante en Nouvelle-France: les écrits de Pierre Potier (1708-1781)}

Caroline Masse, Université du Québec à Montréal

Dans le domaine des études lit- "mettre au jour" les écrits des Canatéraires québécoises, plusieurs cher- diens et des Français d'Amérique, cheurs s'efforcent aujourd'hui de antérieurs au xIx ${ }^{\mathbf{e}}$ siècle canadien. Les 
travaux ${ }^{1}$ de ces "archéologues" du littéraire permettent d'exhumer des textes (correspondances, mémoires, pétitions, pamphlets) et de les rendre accessibles aux chercheurs. C'est pour cette raison que chacun des textes retrouvés est un événement. On ne peut donc que souligner le travail de Robert Toupin, directeur de l'Institut Montserrat de Saint-Jérôme, qui après de nombreuses publications sur les écrits et les aventures des jésuites canadiens ${ }^{2}$ nous offre aujourd'hui un impressionnant travail de transcription et de collation des écrits d'un autre père jésuite, le belge Pierre Potier, "un homme de lettres, épris d'érudition ${ }^{3}$.

Né à Blandain en Belgique, le jésuite Pierre Potier s'embarque comme missionnaire pour Québec, où il arrive le $1^{\text {er }}$ octobre 1743 . Il s'initie durant quelques mois à la langue huronne auprès du père PierreDaniel Richer à la mission de Lorette, puis il quitte le gouvernement de Québec pour la région des Grands Lacs, à la mission huronne de l'île aux Bois-Blancs où il arrive le 25 septembre 1744 . Lorsque la mission est déplacée en 1747 à la Pointe de Montréal ${ }^{4}$, le père Potier y est missionnaire jusqu'à sa mort le 16 juillet 1781.

Le projet de Robert Toupin était de rendre accessible la masse documentaire, en plus de répondre à un certain nombre de questions: sur le plan de la stratégie. pastorale et du projet missionnaire, la tâche du père Potier fut-elle accomplie? Au plan de la culture savante du xviII eiècle, le père Potier représente-t-il un être d'exception, aurait-il dû être - plus qu'un passionné de lecture et d'écriture " (10), •plus qu'un intellectuel affamé d'érudition - (10)? Robert Toupin éprouve la nécessité de justifier son corpus et affirme lui-même que les écrits de père Potier * n'ont pas l'originalité qui caractérise les Relations du xvie siècle. (9). Il nous apparaît qu'il n'est pas tant nécessaire ici de marquer "l'originalité "ou le -caractère exceptionnel "d'un auteur, mais bien de rendre compte des pratiques d'écriture, en plein Siècle des lumières, ici même au Canada.

Comme le dit l'auteur, Pierre Potier est avant tout un encyclopédiste sur le mode mineur, attaché à * cueillir au passage les fruits d'une curiosité - (7), et qui, accumulant de nombreuses relations sur tout ce qu'il voit, lit ou entend, semble pris d'une véritable rage documentaire:
À considérer le dispositif d'une seule petite page, contenant par- fois au-delà de 1000 mots (sur 10 millions environ, dont témoi- gne la documentation), on s'aper- çoit qu'une excellente plume reste - durant un demi-siècle - le dénominateur constant d'une paisible existence qui "épargne. le temps réservé aux choses de l'esprit, même parmi les Hurons des "pays d'en haut n. (23)

Ainsi, Pierre Potier nous livre un portrait sans doute plus fidèle du Canada au $\mathrm{xvIII}^{\mathrm{e}}$ siècle que ne l'avaient fait les jésuites du siècle précédent: il n'écrit pas pour un supérieur en France, mais bien pour luimême, par souci encyclopédique. Les récits du père Potier nous donnent de précieux renseignements, notamment sur la toponymie et le parler des Français d'Amérique au $\mathrm{XVIII}^{\mathrm{e}}$ siècle.

Si Robert Toupin a choisi de se consacrer à la diffusion des écrits de Pierre Potier, c'est parce que ce der- 
nier lui semble représenter un type différent de missionnaire, actif à la fois sur le plan intellectuel et sur le plan pastoral. Il en veut pour preuve la masse documentaire recueillie. À partir de ses cahiers ${ }^{5}$ de notes personnelles, rédigés au fil des jours de sa formation en France et de sa vie en Nouvelle-France, se dévoilent en effet le profil de la formation et le portrait intellectuel de Pierre Potier. À ces cahiers s'ajoute aussi le catalogue de la bibliothèque Potier ${ }^{6}$. Nous avons donc une idée assez juste de l'érudition du missionnaire ${ }^{7}$, ce que Robert Toupin nomme ale réseau enchevêtré de l'érudition de Pierre Potier " (12).

Le livre de Robert Toupin est divisé en deux volumes. Le premier se consacre aux documents qui illustrent la formation intellectuelle de Pierre Potier et son insertion dans la culture savante du xvIII $^{\mathrm{e}}$ siècle: correspondances, autobiographie, journaux de voyage, divers écrits sur la pastorale, sur les coutumes des Amérindiens et, surtout, le document sans doute le plus remarquable, le plus original de toute sa production, Façons de parler et termes français.

Quant au second volume, consacré aux registres de la mission huronne du Détroit et de la paroisse française Notre-Dame de l'Assomption de la Pointe de Montréal, il rend compte de l'activité pastorale soutenue du père Potier. Essentiellement constituée de la transcription des registres de mariages, baptêmes et services funéraires ${ }^{8}$, cette seconde partie intéressera moins les littéraires que les anthropologues et les ethnologues en ce qu'elle donne de précieux renseignements sur l'établissement de la mission. Elle trace également une esquisse de l'organi- sation sociale et politique amérindienne, en plus de fournir des renseignements sur l'évangélisation des Hurons par les missionnaires. Mentionnons également le double recensement de 1747 de la nation huronne (dans le premier volume celui-là), effectué par le père Potier. Il permet de suivre la lente extinction de la nation huronne à la fin du XVIII siècle. Le premier volume est donc plus intéressant d'un point de vue littéraire et c'est essentiellement de celui-ci que nous rendrons compte.

La pièce de résistance des écrits du père Potier est sans aucun doute le document Façons de parler et termes français. Il s'agit là du premier lexique du parler français en Amérique. Le document se présente sous la forme d'un manuscrit composé de notes sur les manières de parler observées par le jésuite depuis son départ de Belgique en 1743 jusqu'à la mission huronne du Détroit (entre 1743 et 1758) ${ }^{9}$. Nous pouvons enfin aujourd'hui apprécier ce projet de longue haleine du père jésuite, car il s'agit là de al'expression tangible d'un projet rédactionnel longuement mûri et lentement articulé " (22), un projet d'écriture mené sur près de quinze années dans lequel le père recense, note et compile tout ce qu'il entend.

Le manuscrit Façons de parler et termes français se compose de plusieurs sections; les premières se présentent sous la forme d' extraits" recopiés par le père Potier dans certains ouvrages (le Dictionnaire de Trévoux, le Dictionnaire de Joubert). Il y a repris les définitions de certains termes français et les définitions de mots, le sens de proverbes et de maximes, etc. ${ }^{10}$ Mais c'est évidemment la section intitulée "termes 
français recueillis çà et là • (355), qui intéressera le plus les chercheurs; voilà la contribution véritablement originale du père Potier. Il s'applique ici à nous transmettre les - manières de dire et de parler. (352). C'est ainsi qu'on savoure les expressions recueillies lors de sa traversée des - Pais-Bas ${ }^{11}$ " (. Les rois Nagent en grande eau: sont dans L'abondance ", 357; *je vous Casserai aux gages: congédierai *, 357), lors du voyage de France vers le Canada ("gentilhomme Degalonné: ruiné , 380 ), au moment de son arrivée à Québec ("Les Poudredries sont accompagnées de froids piquants : eparpillemens de nege •, 381), à Lorette (.Boire le Coup abnakis: grand verre d'eau de vie", 383; "Le Tambour du ventre: La cloche qui appelle à table ", 393), ou encore sur la route de Québec à Détroit et de Détroit à l'île aux BoisBlancs (* La mer est Coquine: attire Les marins", 426; •il rase 50 ans: approche ", 432).

Le journal et les notes de voyage de Pierre Potier occupent un espace considérable. Ces documents possèdent un grand intérêt pour l'étude topographique et la nomenclature des noms de lieux. Ici encore, au carnet de voyage personnel de Pierre Potier (de Blandain jusqu'à son port d'embarquement pour l'Amérique La Rochelle - et de Québec à Détroit) s'ajoutent les journaux recopiés de certains de ses prédécesseurs sur les mêmes sentiers. Le père Potier n'a pas forcément emprunté lui-même toutes ces routes, mais selon une attitude encyclopédique, il note tout ce qui concerne son sujet. La comparaison entre les différents manuscrits permet d'intéressantes découvertes pour les topographes.
Par exemple, en analysant deux récits de voyage recopiés par Potier, on découvre qu'une rivière, appelée - Marie-de-Larme. par le cartographe Duvernet en 1778 , se retrouve sur l'itinéraire d'un certain Charleau ${ }^{12}$. Mais elle s'appelle alors la arivière à Rouquillette, située non loin d'un site prénommé -le Marais de L'Orme. On saisit alors fort bien l'erreur de transcription du cartographe de 1778 et le glissement sémantique qui s'est opéré!

De la correspondance du père Potier, il reste près d'une centaine de lettres qui couvrent la période 17461781 et possèdent un grand nombre de destinataires: religieux ou supérieurs ecclésiastiques, seigneurs de l'époque, commandants militaires. Le livre premier contient également une autobiographie du père Potier, un recensement des villages hurons effectué en 1747, un lexique huronfrançais, des écrits sur les coutumes des Amérindiens, des écrits sur la pastorale, les livres de comptes de la mission huronne et de nombreux autres documents.

Mentionnons aussi deux documents particulièrement intéressants pour l'histoire des pratiques de lecture: le Catalogue de la bibliothèque de Pierre Potier ${ }^{13}$ ainsi que l'Inventaire des effets de P. Potier ${ }^{14}$ qui constituent un matériel précieux pour connaitre les usages de lecture chez les clercs et les membres du clergé à la fin du xvIII siècle canadien. Il faut aussi souligner le travail d'édition: cet ouvrage plus que volumineux est un instrument précis, facile à consulter, grâce entre autres à l'index onomastique, à la liste des abréviations contenues dans les textes de Potier, etc. 
Robert Toupin nous donne accès à cette documentation considérable: les historiens, les anthropologues, les ethnologues, les littéraires, les démographes, les linguistes, tous y trouveront leur compte. Il s'agit d'un ouvrage indispensable à la fois sur l'état des "savoirs" et sur la vie des missions jésuites au XviII ${ }^{\mathrm{e}}$ siècle.

1. Un important travail de défrichement est déjà accompli par les publications de l'équipe du CRELIQ de l'Université Laval. Les travaux du CRELIQ s'inspirent d'une approche institutionnelle de la littérature (les infrastructures, les associations, l'imprimerie, etc.). À l'UQAM, le groupe de recherche Archéologie du littéraire au Québec (ALAQ), dirigé par Bernard Andrès, analyse pour sa part les écrits de ces protoscripteurs d'un point de vue interne, observant l'énonciation et les stratégies argumentatives de ces textes. Pour les travaux de ces deux groupes voir notamment Maurice Lemire et Denis Saint-Jacques (dir.), La vie littéraire au Québec, tome I : La voix française des nouveaux sujets britanniques, 1764-1805, Sainte-Foy, Presses de l'Université Laval, 1991, 498 p.; La vie littéraire au Québec, tome II : Le projet national des Canadiens, 1806-1839, Sainte-Foy, Presses de l'Université Laval, 1992, 587 p.; La vie littéraire au Québec, tome III : Un peuple sans bistoire ni littérature, 1840-1869, SainteFoy, Presses de l'Université Laval, 1996, 671 p. Voir également les travaux de Bernard Andrès, * De l'archive au corpus: problématique d'une archéologie du littéraire au Québec (1764-1815)•, Principes du littéraire au Québec (1766-1815), Montréal, Cahiers de l'ALAQ, $\mathrm{n}^{\circ} 2$, été 1993, p. 9-16. Mentionnons en particulier le numéro spécial que Voix et Images a consacré à l'étude de l'Archéologie du littéraire au Québec ( ${ }^{\circ} 59$, hiver 1995).

2. Voir notamment Robert Toupin, Arpents de neige et Robes noires. Brève relation sur le passage des jésuites en NouvelleFrance, aux $x \mathrm{~V}^{f}$ et $\mathrm{x}$ vif siècles, Montréal, Bellarmin, 1991, $129 \mathrm{p}$.

3. Robert Toupin, Les écrits de Pierre Potier, Ottawa, Les Presses de l'Université d'Ottawa, . Amérique française * $\mathrm{n}^{\circ} 3$, 1996, 1329 p. La citation est extraite de la page 7. Désormais, toute citation tirée de cet ouvrage sera suivie du folio entre parenthèses.

4. Sur le site de l'actuelle ville de Windsor en Ontario.

5. La moitié des cahiers de notes auraient été perdus, selon Robert Toupin. Il reste aujourd'hui trente-huit cahiers manuscrits (notes de cours et de lecture à contenu linguistique, littéraire, scientifique ou pastoral), qui nous indiquent sa formation collégiale et ses intérêts personnels.

6. Voir l'appendice II, p. 1209-1230.

7. Soulignons qu'il est assez exceptionnel, compte tenu de l'époque, de posséder tant de documents ayant appartenu à un missionnaire, leurs conditions de vie étant peu propices à la conservation de leurs biens.

8. Malheureusement aucun des sermons, aucune prédication du père Potier n'est parvenue jusqu'à nous.

9. Ce manuscrit avait fait l'objet d'éditions partielles et avait déjà été mis à la disposition des chercheurs par le biais de l'étude linguistique qu'en a faite Peter $\mathrm{W}$. Halford de l'université de Windsor en 1994. Voir Peter W. Halford, Le français des Canadiens à la veille de la Conquête. Témoignage du père Pierre Pbilippe Potier, s.j., Ottawa, Les Presses de l'Université d'Ottawa, 1994, xi, 380 p.

10. Ces parties sont mentionnées dans l'ouvrage de Robert Toupin, mais elles ne sont pas retranscrites.

11. Dans les extraits cités ci-après, nous respectons l'orthographe du père Potier.

12. Une note nous mentionne que ce texte recopié par Potier serait extrait des gazettes du temps, mais sans plus de précisions (voir Appendice I, p. 1202).

13. Voir appendice II, p. 209.

14. Voir Livre un - Documents Annexes DA8, p. 713-719. 